\title{
The effect of celiac disease on the oral cavity: a
} \section{review}

\begin{abstract}
Celiac disease is characterized by a permanent autoimmune sensitivity to gluten that leads to a disorder of the small intestinal mucosa and affects several organs. Alterations in the oral cavity are frequently observed in patients with celiac disease and may allow for early diagnosis and treatment. Specifically, dental enamel defects, recurrent aphthous Stomatitis, delayed eruption of teeth, and malocclusion have been found to be more common in this patient population. Therefore, dental clinicians should be aware of the oral features of this condition in order to facilitate early diagnosis and prevent consequences that can affect the overall health status of the patient.
\end{abstract}

Keywords: celiac (coeliac) disease, enamel defect, recurrent aphtous stomatitis, delayed tooth eruption, malocclusion
Volume 9 Issue I - 2018

\author{
Majedah A Al Homaidhi \\ Department of Pediatric Dentistry and Orthodontics, King Saud \\ University, Riyadh, Saudi Arabia
}

Correspondence: Majedah A Al-Homaidhi, Department of Pediatric Dentistry and Orthodontics, College of Dentistry, King Saud University, Riyadh, Saudi Arabia, Tel +966-I I-46948I4, Emailmalhomaidhi@ksu.edu.sa

Received: January 14, 2018 | Published: January 25, 2018

\section{Abbreviations: CD, celiac disease; TTG, tissue transglutaminase; Ig, immunoglobulin, RAS, recurrent aphthous stomatitis}

\section{Introduction}

Celiac disease is one of the most common chronic systemic autoimmune disorders. It is triggered by the ingestion of gluten, which is a protein found in wheat, rye, and barley. ${ }^{1}$ Only a part of the gluten complex plays a significant role in the pathogenesis of Celiac disease; this includes gliadin in wheat, secalin in rye, hordein in barley, and avenin in oats. ${ }^{2}$ Celiac disease generally presents after the sixth month of life, when gluten is normally introduced in the human diet. ${ }^{3,4}$ In humans, gluten is not completely digested; it is only broken down into peptides, which are transferred across the intestinal wall and modified by tissue transglutaminase (TTG). TTG is generally present in an inactivated form, and the cause of its activation in Celiac disease remains unknown. TTG activation leads to the production of negatively charged peptide molecules, which complement the positively charged structures of HLA DQ2 and HLA DQ8 antigens. Subsequently, escalation of the immune response results in the production of specific autoantibodies, which are the bases of disease diagnostics. This process results in an inflammatory state in the intestinal wall and consequent damage to the intestinal villi, which affects the absorption process and leads to progressing malnutrition and related consequences. ${ }^{5}$ Celiac disease can be typical or atypical. The typical (classic) type develops when gluten is introduced in the diet, usually between 6 and 24 months of age. Typical features include insufficient height and weight and gastrointestinal symptoms such as abdominal bloating and pain, vomiting, constipation, and diarrhea. The atypical type, on the other hand, affects older children and adults. Celiac disease is diagnosed on the basis of clinical symptoms; serological tests for the detection of serum immunoglobulin (Ig) A (IgA), TTG antibody, and IgA-endomysial antibody; and histopathological examination of biopsy specimens from the intestinal mucosa. ${ }^{6,7}$ Recently, genetic studies identifying HLA DQ2/ DQ8 antigens have been utilized more frequently, ${ }^{8}$ because the spontaneous disappearance of antibodies over time has been observed in a proportion of children with Celiac disease. ${ }^{9}$ Approximately $83 \%$ patients remain undiagnosed or are misdiagnosed, which can result in irreversible medical and dental complications. ${ }^{10}$ Celiac disease is a multifactorial disorder, with both genetic and environmental factors playing a role in the etiology. ${ }^{11}$ Genetically close relatives of a patient with celiac disease are at significant risk of developing the disorder $(5 \%-15 \%){ }^{12}$ In addition, patients with autoimmune disorders such as type 1 diabetes mellitus, Williams syndrome, IgA deficiency, autoimmune thyroiditis, and Down syndrome, carry a higher risk of Celiac disease development. ${ }^{13}$ Among environmental factors, the most important one is early introduction of gluten in a child's diet. In fact, the disease can be prevented by early introduction of gluten in a child's diet to build the immune tolerance. ${ }^{14}$ The current dietetic recommendations advise that gluten should be gradually introduced in the diet between 4 and 6 months of age, with careful observation for undesired reactions. Late introduction of gluten may result in atypical Celiac disease, with delayed diagnosis or undiagnosed disease for several years. ${ }^{11}$ Alterations in the oral cavity are frequently observed in patients with Celiac disease and may allow for early diagnosis and treatment. Specifically, dental enamel defects, recurrent aphthous stomatitis (RAS), delayed eruption of teeth, and malocclusion have been found to be more common in this patient population. Awareness of Celiac disease among health professionals remains poor, and delays in diagnosis are common. Therefore, as clinicians, it is very important to be aware of the potential presentations, particularly in the oral cavity, in order to facilitate early diagnosis and prevent consequences that can affect the overall health status of the patient.

\section{Discussion}

\section{Prevalence}

Several studies have been conducted to evaluate the prevalence of Celiac disease in the general population, which is usually estimated by counting the number of diagnosed Celiac disease patients detected by serological screening of a population sample. The prevalence of Celiac disease is $2 \%-3 \%$ in Finland and Sweden, $0.2 \%$ in Germany, ${ }^{15}$ $1.6 \%$ in Asia, ${ }^{16}$ and $1 \%$ in the United States. ${ }^{17}$ The prevalence was $4 \%$ in a recent study by Cilleruelo et al. ${ }^{9}$ who used the HLA-DQ2 test. ${ }^{9}$ This variability in the worldwide prevalence can be attributed to differences in the type of test used for screening, sample size, age, and criteria for celiac disease diagnosis among studies. ${ }^{18,19}$ A strong 
association between Celiac disease and autoimmune conditions has been reported..$^{20-22}$ In a systemic review conducted by Dube the prevalence of celiac disease in at-risk populations was as follows: $3 \%-6 \%$ in patients with type 1 diabetes, up to $20 \%$ in first-degree relatives of patients with celiac disease, $10 \%-15 \%$ in patients with symptomatic iron-deficiency anemia, $3 \%-6 \%$ in patients with asymptomatic iron-deficiency anemia, and $1 \%-3 \%$ in patients with osteoporosis. $^{23}$

\section{Signs and symptoms}

celiac disease is a complex disease associated with several extra intestinal signs and symptoms such as short stature, growth failure, weight loss, osteoporosis, anemia resistant to iron treatment, arthritis, increased liver enzymes, itchy skin rash (dermatitis herpetiformis), numbness, chronic fatigue, depression, delayed puberty, infertility, and schizophrenia. ${ }^{24,25}$ Decreased bone density has been found in approximately $75 \%$ of newly diagnosed patients, with a $40 \%$ greater risk for bone fracture. ${ }^{2}$ In addition to the systemic problems mentioned above, other pathological changes within the oral cavity, including enamel mineralization defects, oral aphtous ulcers, and malocclusion, have been identified..$^{27-29}$ Delayed tooth eruption, atrophic glossitis, geographic tongue, and burning sensation, particularly in the tongue, may also appear during the course of celiac disease. ${ }^{24-30}$

\section{Oral manifestations}

Prolonged exposure to gluten and delayed diagnosis of celiac disease increase the risk of pathologies that may affect both the developing dentition and the oral mucosa. The oral features of celiac disease are varied and atypical, and affected patients may exhibit enamel defects, delayed tooth eruption, and impaired mandibular jaw growth. ${ }^{27}$ Among the oral manifestations of celiac disease, enamel defects and RAS are the most common and well documented in several studies (Table 1). ${ }^{29,31-40}$

Table I Prevalence of dental developmental defects and RAS in patients with $C D$

\begin{tabular}{lllll}
\hline Studies & Year & Sample size (CD vs. normal) & Enamel defects (\%) (CD vs. normal) & RAS (\%) (CD vs. normal) \\
\hline Priovolou et al. $^{31}$ & 2004 & 27 vs. 27 & 83.3 vs. 50 & ---- \\
${\text { Bucci et al. }{ }^{37}}^{2006}$ & 72 vs. 162 & 20 vs. 5.6 & 33.3 vs. 23.4 \\
Campisi et al. $^{29}$ & 2007 & 197 vs. 413 & 33 vs. 9 & 42 vs. 2 \\
Avsar \& Kalayci $^{39}$ & 2008 & 64 vs. 64 & 42.2 vs. 9.4 & ---- \\
Acar et al. ${ }^{40}$ & 2012 & 35 vs. 35 & 40 vs. 0 & 37.1 vs. II.4 \\
Cantekin et al. ${ }^{38}$ & 2015 & 25 vs. 25 & 48 vs. 16 & 44 vs. 0 \\
Dane \& Gurbuz & 2016 & 35 vs. 35 & 54.3 vs. 20 & 31.4 vs. 0 \\
Saraceno et al. ${ }^{54}$ & 2016 & 166 vs 166 & 76 vs. 65 & 69 vs. 43 \\
\hline
\end{tabular}

RAS Recurrent Aphthous Stomatitis, CD: Celiac Disease

\section{Enamel defects}

Genetic, systemic, or local injuries during the different stages of dental organ development can result in defective enamel formation. Altered matrix formation results in enamel hypoplasia, which is quantitative enamel defect ${ }^{41}$ On the other hand, injuries during the maturation phase lead to areas of hypo calcification, which is a qualitative enamel defect ${ }^{42}$ The maturation process in all primary teeth begins in the fourth month of prenatal development, whereas the mineralization of permanent teeth begins as early as the prenatal period (first molars) up to 30-36 months of age (second molars; (Table 2)). ${ }^{43}$
Therefore, any systemic or local injury that occurs during the period of tooth mineralization may result in enamel abnormalities. ${ }^{41,42}$ The precise mechanism underlying the development of enamel defects in celiac disease patients remains unclear, although several hypotheses have been suggested. The most well-accepted mechanism is immunemediated damage, ${ }^{44}$ followed by stimulation of lymphocytes in the oral cavity by gluten. ${ }^{45}$ Munoz et al. reported that anti-gliadin antibodies, which are present in celiac disease patients, may react with enamel matrix proteins (amelogenin and ameloblastin) ${ }^{46}$ Nutritional disturbances due to mal absorption are also considered to play a role. ${ }^{47}$

Table 2 Dental growth and development of the permanent dentition

\begin{tabular}{|c|c|c|}
\hline Tooth & Calcification begins at & Crown (enamel) completed at \\
\hline Central incisors & $3-4$ months & $4-5$ years \\
\hline \multirow{2}{*}{ Lateral incisors } & Maxilla: 10-12 months & $4-5$ years \\
\hline & Mandible: 3-4 months & $4-5$ years \\
\hline Canines & $4-5$ months & $6-7$ years \\
\hline First premolars & I8-24 months & $5-6$ years \\
\hline Second premolars & $24-30$ months & $6-7$ years \\
\hline First molars & Birth & $30-36$ months \\
\hline Second premolars & $30-36$ months & $7-8$ years \\
\hline \multirow{2}{*}{ Third molars } & Maxilla: 7-9 years & \\
\hline & Mandible: 8-10 years & \\
\hline
\end{tabular}

Table adapted from the American Academy of Pediatric Dentistry ${ }^{43}$ 
The overall prevalence of dental enamel defects in celiac disease patients with a primary dentition is $5.8 \%$ to $13.3 \%$, while that in celiac disease patients with a permanent dentition ranges from $9.5 \%$ to $95.9 \%{ }^{44}$ This difference between dentitions can be attributed to the fact that the permanent teeth develop during the early months of life, after the introduction of gluten in the diet, while primary teeth develop before birth. Therefore, enamel defects are more common in patients who develop celiac disease before the age of 7 years, whereas they are not frequently observed in adults because of late celiac disease development or the treatment or extraction of the affected teeth. ${ }^{30,39}$ In fact, enamel defects have been associated with celiac disease and could be the sole sign of celiac disease in children. ${ }^{12-48}$ Bossu et al. ${ }^{32}$ evaluated enamel defects in patients with celiac disease under a scanning electron microscope and found alterations in the shape and size of the enamel prisms (shorter and irregular enamel prisms with a decreased interprismatic substance), which corresponded to damage that occurred during the early phases of tooth crown development during the first year of life. In other studies, the amount of calcium and phosphorous was lower in hypo mineralized areas of enamel than in normal enamel..$^{49}$ Because the clinical picture of dental enamel defects in CELIAC DISEASE patients may be diverse, a four-grade classification proposed by Aine et al. ${ }^{48}$ is widely used for the purpose of assessment (Table 3 ). ${ }^{48}$

Table 3 Classification of dental enamel defects ${ }^{48}$

\section{Grade Manifestation}

Defects in color: single or multiple opacities; cream, yellow, or brown in color; well-defined or diffuse margins; involvement of one surface of the crown

Mild structural defects: rough enamel surface with horizontal grooves and superficial pits; possible mild opacities and discolorations; involvement of one surface of the crown or the entire crown

Evident structural defects: One surface of the crown or the entire crown is rough or covered with grooves of varying depths and widths or large and deep pits. Discolorations and opacities of different colors can be present.

Severe structural defects: The shape of the tooth is changed, with sharp cusps and incisive margins that are irregularly thin and rough. Enamel thinning is generalized, and the edge of the defect is well defined. The lesions can be hardly discolored.

According to these authors, enamel defects in celiac disease patients should be present symmetrically and chronologically in the same anatomical groups (specific) of teeth in all quadrants of the dentition. ${ }^{48}$ In contrast, enamel defects in healthy patients are nonspecific..$^{50,29,31,36-38}$ Most enamel defects in celiac disease patients have been reported to be mild and classified as Grade I defects according to the classification proposed by Aine et al. ${ }^{29-31,36,39,48}$ Costacurta et al..$^{30}$ examined the presence of enamel defects in 300 celiac disease patients (mean age, $8.16 \pm 2.95$ ) and found that the canines $(45 \%)$ and second molars $(51 \%)$ were the most commonly involved deciduous teeth, while the central incisors $(25 \%)$, lateral incisors $(20 \%)$, and first molars $(24 \%)$ were the most commonly involved permanent teeth, followed by the canines $(9 \%)$, first premolars $(8 \%)$, second premolars $(7 \%)$, and second molars $(7 \%) .{ }^{30}$

\section{RAS}

RAS in patients with celiac disease is characterized by the presence of multiple small ulcers or a single large ulcer in the oral cavity. ${ }^{29,37}$ The precise mechanism and aetiology of RAS in celiac disease patients is not clear. However, several mechanisms have been hypothesized, such as iron, folic acid, or vitamin B12 deficiency as a result of malabsorption in CELIAC DISEASE patients who do not adhere to a gluten-free diet. $^{51}$ Several studies have associated hematinic deficiency with RAS, with varying prevalence among populations because of varying genetic backgrounds and dietary habits. ${ }^{52,53}$ The prevalence of RAS in patients with untreated celiac disease has been reported to range from $33 \%$ to $69 \%$, with the highest prevalence in children, adolescents, and women. ${ }^{29,36-38,54}$

\section{Other oral manifestations}

Although celiac disease can result in several oral manifestations, not all are as common as enamel defects and RAS. Relatively uncommon manifestations include erythema, atrophic glossitis, burning sensation (particularly in the tongue), dryness, delayed eruption, and malocclusion. ${ }^{27,38,55} \mathrm{~A}$ few studies have investigated delayed tooth eruption, which could be a result of malnutrition associated with celiac disease, and found a prevalence of $20 \%$ to $27 \% .29,30,56$ However, Mina et al..$^{57}$ did not observe any delay in tooth eruption in celiac disease patients. ${ }^{57}$ Malocclusion is frequent in celiac disease patients. Abdul-Wahid et al.$^{58}$ found a prevalence of $39.18 \%$ in celiac disease patients.58 Malocclusion in celiac disease patients could be a result of impaired maxillary and mandibular growth and delayed tooth eruption..$^{27}$ Federico et al..$^{59}$ studied the effects of a gluten-free diet on growth factors in patients with celiac disease and found that multiple alterations in the growth axis observed during the active phase of the disease disappeared during the gluten-free diet. ${ }^{59}$ With regard to caries, the frequency is lower in celiac disease patients than in healthy individuals, probably because of better diet control in the former. ${ }^{31,44,54,60}$ In contrast to these findings, some studies have reported a higher frequency of caries in the permanent dentition of celiac disease patients. ${ }^{30,38,39}$ Some studies have evaluated the effects of celiac disease on the salivary parameters of affected patients. Acar et al. $^{40}$ evaluated the salivary parameters of patient with celiac disease and compared the findings with those for healthy individuals; they found a lower prevalence of salivary mutans streptococci and lactobacilli colonization in celiac disease patients than in healthy individuals, with no significant differences in other parameters such as the salivary flow rate, $\mathrm{pH}$, and buffering capacity. ${ }^{40}$ In contrast, Tian et al ${ }^{61}$ found a higher prevalence of lactobacilli with a higher glutenase activity in celiac disease patients, which may play a role in the pathogenicity of the disease. ${ }^{61}$ Dane and Gurbuz found a lower salivary flow rate and buffering capacity in celiac disease patients. ${ }^{36}$

\section{Conclusion}

The complications, signs, and symptoms of celiac disease can be easily and effectively treated by the lifelong maintenance of a glutenfree diet if it is diagnosed early in life. However, a gluten-free diet should not be started before a biopsy, because the diet will result in healing of the intestinal lesions and make diagnosis difficult. Dentists (particularly general practitioners and pedodontists) could be the first ones to diagnose celiac disease by meticulous observation of the oral cavity for the presence of specific dental enamel defects, RAS, malocclusion, and delayed tooth eruption. If these signs are present, 
the dentist should consider questioning the patient about other clinical symptoms such as abdominal pain, abdominal swelling, diarrhea, weight loss, poor growth, anemia, and family history of the disease.

\section{Funding}

None.

\section{Acknowledgements}

None.

\section{Conflicts of interest}

The authors declare that there is no conflict of interest.

\section{References}

1. Lauret E, Rodrigo L. Celiac disease and autoimmune-associated conditions. Biomed Res Int. 2013:127589.

2. Marsh MN. The small intestine: mechanisms of local immunity and gluten sensitivity. Clin Sci (Lond). 1981;61(5):497-503.

3. Mearin ML. Celiac disease among children and adolescents. Curr Probl Pediatr Adolesc Health Care. 2007;37(3):86-105.

4. Da Silva PC, De Almeida PDV, Machado MAN, et al. Oral manifestations of celiac disease. A case report and review of the literature. Med Oral Patol Oral Cir Bucal. 2008;13(9):E559-E562.

5. Vogelsang $\mathrm{H}$, Schwarzenhofer $\mathrm{M}$, Steiner $\mathrm{B}$,et al. In vivo and in vitro permeability in coeliac disease. Aliment Pharmacol Ther. 2001;15(9):1417-1425.

6. Picarelli A, Maiuri L, Frate A, et al. Production of antiendomysial antibodies after in-vitro gliadin challenge of small intestine biopsy samples from patients with coeliac disease. Lancet. 1996;348(9034):1065-1067.

7. Russo PA, Chartrand LJ, Seidman E. Comparative analysis of serologic screening tests for the initial diagnosis of celiac disease. Pediatrics. 1999;104(1 Pt 1):75-78.

8. Dickey W, Hughes DF, McMillan SA. Disappearance of endomysial antibodies in treated celiac disease does not indicate histological recovery. Am J Gastroenterol. 2000;95(3):712-714.

9. Cilleruelo ML, Fernández-Fernández S, Jiménez-Jiménez J, et al Prevalence and natural history of celiac disease in a cohort of at-risk children. J Pediatr Gastroenterol Nutr. 2016;62(5):739-745.

10. Farrell RJ, Kelly CP. Celiac sprue. N Engl J Med. 2002;346(3):180-188.

11. Rivera E, Assiri A, Guandalini S. Celiac disease. Oral Dis 2013;19(7):635-641.

12. Hill ID, Dirks MH, Liptak GS, et al. Guideline for the diagnosis and treatment of celiac disease in children: recommendations of the North American Society for Pediatric Gastroenterology, Hepatology and Nutrition. J Pediatr Gastroenterol Nutr. 2005;40(1):1-19.

13. AGA Institute MF. AGA Institute Medical Position Statement on the Diagnosis and Management of Celiac Disease. Gastroenterology. 2006;131(6):1977-1980.

14. Armstrong MJ, Hegade VS, Robins G. Advances in coeliac disease. Curr Opin Gastroenterol. 2014;28(2):104-112.

15. Mustalahti K, Catassi C, Reunanen A, et al. The prevalence of celiac disease in Europe: Results of a centralized, international mass screening project. Ann Med. 2010;42(8):587-595.

16. Singh P, Arora S, Singh A, et al. Prevalence of celiac disease in Asia: A systematic review and meta-analysis. $J$ Gastroenterol Hepatol. 2016;1(6):1095-1101.
17. Fasano A, Berti I, Gerarduzzi T, et al. Prevalence of celiac disease in atrisk and not-at-risk groups in the United States: a large multicenter study. Arch Intern Med. 2003;163(3):286-292.

18. Uibo O, Teesalu K, Metskula K, et al. Screening for celiac disease in Down's syndrome patients revealed cases of subtotal villous atrophy without typical for celiac disease HLA-DQ and tissue transglutaminase antibodies. World J Gastroenterol. 2006;12(9):1430-1434.

19. Carlsson A, Axelsson I, Borulf S, et al. Prevalence of IgA-antigliadin antibodies and IgA-antiendomysium antibodies related to celiac disease in children with Down syndrome. Pediatrics. 1998;101(2):272-275.

20. Craig ME, Prinz N, Boyle CT, et al. Response to comment on Craig et al. Prevalence of celiac Disease in 52,721 youth with type 1 diabetes: international comparison across three continents. Diabetes Care. 2017;40(11):e168-e169.

21. Alshareef MA, Aljabri KS, Bokhari SA, et al. The prevalence of celiac disease in Saudi patients with type 1 diabetes mellitus: Cross sectional study. 2016;1(1):2-5.

22. Grace-Farfaglia P. Bones of contention: Bone mineral density recovery in celiac disease-A systematic review. Nutrients. 2015;7(5): 3347-3369.

23. Dubé C, Rostom A, Sy R, et al. The prevalence of celiac disease in average-risk and at-risk Western European populations: a systematic review. Gastroenterology. 2005;128(4 Suppl 1):S57-S67.

24. Krzywicka B, Herman K, Kowalczyk-Zajac M, et al. Celiac disease and its impact on the oral health status - Review of the literature. Adv Clin Exp Med. 2014;23(5):675-681.

25. Wijarnpreecha K, Jaruvongvanich V, Cheungpasitporn W, et al Association between celiac disease and schizophrenia: a meta-analysis. Eur J Gastroenterol Hepatol; 2017.

26. Lucendo AJ, García-Manzanares A. Bone mineral density in adult coeliac disease: an updated review. Rev Esp Enferm Dig. 2013;105(3):154-162.

27. Bilello G, Ciulla C, Caradonna C. Celiac disease and malocclusion. Recenti Prog Med. 2010;101(4):159-162.

28. Ferraz EG, Campos EDJ, Sarmentó VA, et al. The oral manifestations of celiac disease: information for the pediatric dentist. Pediatr Dent. 2012;34(7):485-488.

29. Campisi G, Di Liberto C, Iacono G, et al. Oral pathology in untreated coelic disease. Aliment Pharmacol Ther. 2007;26(11-12): 1529-1536.

30. Costacurta M, Maturo P, Bartolino M, et al. Oral manifestations of coeliac disease: A clinical-statistic study. Oral Implantol (Rome). 2010;3(1):12-19.

31. Priovolou CH, Vanderas AP, Papagiannoulis L. A comparative study on the prevalence of enamel defects and dental caries in children and adolescents with and without coeliac disease. Eur $J$ Paediatr Dent. 2004;5(2):102-106.

32. Bossù M, Bartoli A, Orsini G, et al. Enamel hypoplasia in coeliac children: a potential clinical marker of early diagnosis. Eur J Paediatr Dent. 2007;8(1):31-37.

33. Cheng J, Malahias T, Brar P, et al. The association between celiac disease, dental enamel defects, and aphthous ulcers in a United States cohort. $J$ Clin Gastroenterol. 2010;44(3):191-194.

34. Jajam M, Bozzolo P, Niklander S. Oral manifestations of gastrointestinal disorders. J Clin Exp Dent. 2017;9(10):e1242-e1248.

35. Amato M, Zingone F, Caggiano M, et al. Tooth wear is frequent in adult patients with celiac disease. Nutrients. 2017;9(12): E1321.

36. Dane A, Gürbüz T. Clinical evaluation of specific oral and salivary findings of coeliac disease in eastern Turkish paediatric patients. Eur $J$ Paediatr Dent. 2016;17(1):53-56. 
37. Bucci P, Carile F, Sangianantoni A, et al. Oral aphthous ulcers and dental enamel defects in children with coeliac disease Acta Paediatr. 2006;95(2):203-207.

38. Cantekin K, Arslan D, Delikan E. Presence and distribution of denta enamel defects, recurrent aphthous lesions and dental caries in children with celiac disease. Pakistan J Med Sci. 2015;31(3):606-609.

39. Avşar A, Kalayci AG. The presence and distribution of dental ename defects and caries in children with celiac disease. Turk $J$ Pediatr. 2008;50(1):45-50.

40. Acar S, Yetkiner AA, Ersin N, et al. Oral findings and salivary parameters in children with celiac disease: a preliminary study. Med Princ $\mathrm{Pr}$. 2012;21:129-133.

41. Seow WK. Enamel hypoplasia in the primary dentition: a review. ASDC J Dent Child. 1991;58(6):441-452.

42. Elhennawy K, Manton DJ, Crombie F, et al. Structural, mechanical and chemical evaluation of molar-incisor hypomineralization-affected enamel: A systematic review. Arch Oral Biol. 2017;83:272-281.

43. American Academy of Pediatric Dentistry. Dental Growth and Developement. Pediatr Dent. 2016. 38(6): 413.

44. Pastore L, Carroccio A, Compilato D, et al. Oral manifestations of celiac disease. J Clin Gastroenterol. 2008;42(3):224-232.

45. Fraser D, Nikiforuk G. The etiology of enamel hypoplasia in children-a unifying concept. J Int Assoc Dent Child. 1982;13(1):1-11.

46. Muñoz F, Del Río N, Sóñora C, et al. Enamel defects associated with coeliac disease: putative role of antibodies against gliadin in pathogenesis. Eur J Oral Sci. 2012;120(2):104-112.

47. Somech R, Spirer Z. Celiac disease: extraintestinal manifestations, associated diseases, and complications. Adv Pediatr. 2002;49:191-201.

48. Aine L, Mäki M, Collin P, et al. Dental enamel defects in celiac disease. $J$ Oral Pathol Med. 1990;19(6):241-245.

49. Kanchan T, Machado M, Rao A, et al. Enamel hypoplasia and its role in identification of individuals: A review of literature. Indian J Dent. 2015;6(2):99-102.
50. Wierink CD, Van Diermen DE, Aartman IHA, et al. Dental enamel defects in children with coeliac disease. Int J Paediatr Dent. 2007;17(3):163-168.

51. Preeti L, Magesh K, Rajkumar K, et al. Recurrent aphthous stomatitis. $J$ Oral Maxillofac Pathol. 2011;15(3):252-256.

52. Scully C, Porter S. Oral mucosal disease: Recurrent aphthous stomatitis. Br J Oral Maxillofac Surg. 2008;46(3):198-206.

53. Natah SS, Konttinen YT, Enattah NS, et al. Recurrent aphthous ulcers today: a review of the growing knowledge. Int J Oral Maxillofac Surg. 2004;33(3):221-234.

54. Saraceno R, Perugia C, Ventura A, et al. Aphthous, celiac disease and other dental disorders in childhood. G Ital Dermatol Venereol. 2016;151(3):239-243.

55. Pastore L, Lo Muzio L, Serpico R. Atrophic glossitis leading to the diagnosis of celiac disease. $N$ Engl J Med. 2007;356(24): 2547.

56. Condò R, Costacurta M, Maturo P, et al. The dental age in the child with coeliac disease. Eur J Paediatr Dent. 2011;12(3):184-188.

57. Mina SS, Azcurra AI, Dorronsoro S, et al. Alterations of the oral ecosystem in children with celiac disease. Acta Odontol Latinoam. 2008;21(2):121-126.

58. Abdul-Wahid SF, Al-Azawi LA, Sc BDSM, et al. Enamel defects and malocclusion in patients with celiac disease. $J$ Bagh Coll Dent. 2005;17(173):98-100

59. Federico G, Favilli T, Cinquanta L, et al. Effect of celiac disease and gluten-free diet on growth hormone-binding protein, insulin-like growth factor-I, and insulin-like growth factor-binding proteins. Horm Res. 1997;48(3):108-114.

60. Farmakis E, Puntis JW, Toumba KJ. Enamel defects in children with coeliac disease. Eur J Paediatr Dent. 2005;6(3):129-132.

61. Tian N, Faller L, Leffler DA, et al. Salivary gluten degradation and oral microbial profiles in healthy individuals and celiac disease patients. Appl Environ Microbiol. 2017;83(6):e03330-e0333016. 